\title{
Burkitt's Lymphoma Presenting as Late-Onset Posttransplant Lymphoproliferative Disorder following Kidney and Pancreas Transplantation: Case Report and Review of the Literature
}

\author{
Seema Naik ${ }^{a}$ Kristy Tayapongsak ${ }^{c}$ Katherine Robbins $^{b}$ \\ Cyrus Koresh Manavi $^{b} \quad$ Mark J. Pettenati $^{d}$ David D. Grier \\ a Blood and Marrow Transplantation, Division of Hematology and Oncology, and \\ ${ }^{b}$ Department of Pathology, Wake Forest Baptist Medical Center, ${ }^{\mathrm{C}}$ Wake Forest \\ University School of Medicine, and dDepartment of Molecular Genetics, \\ Wake Forest University School of Medicine, Winston-Salem, N.C., USA
}

\section{Key Words}

Burkitt's lymphoma - Epstein-Barr virus - Posttransplant lymphoproliferative disorders . Immunosuppression $\cdot$ B-cell lymphoma 2

\begin{abstract}
Posttransplant lymphoproliferative disorders (PTLD) are a rare, but serious complication following transplantation. Late-onset PTLD are often associated with more monoclonal lesions and consequently have a worse prognosis. There are only isolated case reports of Burkitt's lymphoma presenting as PTLD. We present an extremely rare, aggressive Burkitt's lymphoma years after kidney and pancreas transplantation which was successfully treated with combination chemotherapy along with withdrawal of immunosuppression. The patient remains in complete remission more than 2 years after his diagnosis. We also provide a succinct review of treatment of various PTLD and discuss the role of Epstein-Barr virus infection in the pathogenesis of PTLD.
\end{abstract}

\section{Introduction}

Posttransplant lymphoproliferative disorders (PTLD) are a rare, but serious complication following transplantation. Occurring exclusively in iatrogenically 
immunosuppressed patients, there is an overall incidence of 1-5\% in solid organ transplant (SOT) recipients and 1\% in hematopoietic stem cell transplant (HSCT) recipients [1]. Most cases of PTLD occur within the first year after transplantation. Late-onset PTLD, as seen in our patient 8 years after transplantation, is often associated with more monoclonal lesions and consequently have a worse prognosis. The term PTLD represents a spectrum of B-cell hyperproliferative states that can be classified as early lesions and polymorphic and monomorphic PTLD, all of which are associated with Epstein-Barr virus (EBV) [2]. Monomorphic PTLD is further divided into Burkitt's lymphoma/Burkitt's-like lymphoma, diffuse large B-cell lymphoma (DLBCL) and classic Hodgkin's-like lymphoma [2].

The occurrence of PTLD in solid organ allograft recipients can be quite varied in clinical presentation, histopathological characteristics and frequency. A variety of lymphomas can develop as PTLD, although Burkitt's lymphoma appears infrequently and remains poorly understood in this clinical setting. In this report, we describe a case of Burkitt's lymphoma presenting as PTLD following kidney and pancreas transplantation. The recipient was 39 years old and displayed gastrointestinal involvement by Burkitt's lymphoma several years following transplant. The tumor displayed the typical histological features of Burkitt's lymphoma but was negative for EBV. The tumor required aggressive chemotherapy and a cessation of immunosuppressive therapy. This report demonstrates that Burkitt's-type lymphomas can develop in the posttransplant setting and that these tumors contain morphologic, cytofluorographic and molecular features identical to Burkitt's lymphomas that occur in non-transplant patients. We would recommend PTLD-Burkitt's lymphomas behave aggressively and require intensive chemotherapeutic intervention [2].

\section{Case Presentation}

Our patient is a 39-year-old man with an extensive past medical history including diabetes mellitus, below knee amputation in 2007, end-stage renal disease, and simultaneous kidney and pancreas transplantation in 2003. He was admitted in July 2010, with the right lower extremity stump infection. His course was complicated by respiratory failure requiring intubation and mechanical ventilation. The patient went home and developed nausea, vomiting, and diarrhea with right lower quadrant pain. He also then noticed a mass in the right side of the neck. He had a right cervical submandibular mass and fullness in the right upper quadrant of his abdomen. He denied any constitutional symptoms, any obstructive symptoms, weight loss or gastrointestinal bleeding. He was on insulin as needed for diabetes following pancreas transplantation. He presented with a right submandibular swelling $3 \mathrm{~cm}$ in diameter, with a healing ulcerative lesion without any tenderness. His physical examination showed stable vital signs: temperature: $97.4^{\circ} \mathrm{F}$; blood pressure: $136 / 76 \mathrm{~mm}$ Hg; pulse: 95 beats per min; weight: 206.7 pounds; weight loss: -7.30 pounds. His abdomen was soft and non-tender. He had no hepatosplenomegaly, no bleeding, no surgical scars and no other cervical, axillary or inguinal lymphadenopathy. The computed tomography (CT) on 08/16/2010 showed a soft tissue mass measuring $11 \times 10 \mathrm{~cm}$ in the right lower quadrant region.

The patient had a needle-guided biopsy of the mass which showed diagnosis of Burkitt's lymphoma with $\mathrm{t}(8 ; 14)$. Pathology from fine needle aspiration showed medium-sized cells with speckled chromatin and multiple small conspicuous nucleoli ( fig. 1). Molecular cytogenetic analysis (fluorescence in situ hybridization) revealed a significant number of MYC/IgH fusion events (fig. 2). Immunohistochemistry showed that the tumor cells were strongly positive for CD20, consistent with mature B-cell lineage (fig. 3 ). The tumor cells showed weak expression of BCL-2 (fig, 4 ). Ki-67 proliferation index was $100 \%$ by Mib-1 immunohistochemistry, consistent with the diagnosis of Burkitt's lymphoma (

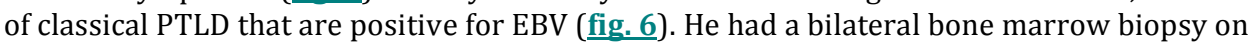


$08 / 26 / 2010$, and then underwent staging workup including neck, chest, abdominal and pelvic CT on $08 / 27 / 2010$. He received treatment as per Burkitt's protocol along with aggressive hydration and was monitored closely for tumor lysis syndrome. He was placed on allopurinol for prophylaxis prior to starting chemotherapy. He did not develop any complications including perforation or tumor lysis. He had partial bowel obstruction at the time of diagnosis, which improved over the period of a few days. He then began course 2 of the protocol with methotrexate on 09/05/2010. Chest X-ray showed no pleural effusion. He had no ascites. He received rituximab on days 8, 10 and 12 . He tolerated and cleared his high-dose methotrexate well.

The patient was diagnosed with Burkitt's lymphoma involving the right submandibular gland and the right ileocolic mass as well. He had presented initially with partial bowel obstruction. The symptoms of bowel obstruction improved with conservative management. The patient was started on chemotherapy in view of the diagnosis of Burkitt's lymphoma. His immunosuppression was maintained in view of chemotherapy and the use of prednisone during Burkitt's protocol. The patient tolerated chemotherapy well and had marked reduction in the abdominal and right submandibular masses. The patient received 6 cycles of chemotherapy which involved high-dose methotrexate, rituximab, doxorubicin and vincristine. He also received intrathecal therapy. He did not receive any additional immunosuppression until completion of all chemotherapy. Mycophenolate mofetil and tacrolimus were maintained in view of continued immunosuppression with chemotherapy. Repeat CT and PET scans after completion of 2 cycles of chemotherapy and at completion of all planned cycles of chemotherapy showed that our patient had no evidence of hypermetabolic disease and continues to be in remission more than 2 years after his original diagnosis.

\section{Discussion}

The majority of PTLD cases are induced by EBV, a gammaherpesvirus. EBV selectively infects B cells, remaining latent in the memory B-cell reservoir for the duration of the host's life. The main viral genes involved in the transformation and persistence of infected B cells are LMP1 and LMP2a. Through self-aggregation on the surface of the infected B cell, LMP2a and LMP1 provide tonic signals leading to the proliferation of transformed memory B cells that remain in the host for life [3]. LMP1 and other EBV proteins such as EBNA 2, are highly antigenic, marking the infected B cells for destruction by cytotoxic T cells (CTLs) in the healthy individual. However, in transplant recipients undergoing immunosuppression, CTLs are immobilized, allowing for the proliferation and immortalization of infected B cells [3]. Posttransplant immunosuppression in HCST recipients typically lasts for 6 months. SOT patients, on the other hand, are immunosuppressed for life in order to ensure continuous proper function of the transplanted organ. In SOT patients, lymphoproliferation of transformed cells are more commonly of recipient origin. A variety of lymphomas can develop as PTLD, although some types appear infrequently and remain poorly understood. PTLDBurkitt's lymphomas behave aggressively and require intensive chemotherapeutic intervention [3]. The tumors display the typical histological features of Burkitt's lymphoma and are markedly positive for EBV. Weak BCL2 expression is still compatible with the diagnosis of Burkitt's lymphoma [4]. Contrary to common belief, strong BCL2 IHC expression is possible in typical Burkitt's lymphoma in adults and cannot be absolutely relied upon to distinguish between Burkitt's lymphoma and DLBCL [5]. Furthermore, the combination of a strong expression of bcl-2 and EBV-positive status of the tumor found in our DLBCL patient suggests the disruption of an apoptotic pathway by EBV, promoting survival signals of the infected B cells [5]. Our Burkitt'sPTLD case had unique clinical and pathological characteristics that may challenge the clinical and pathological diagnosis and need to be recognized and managed in a timely manner. The patient presented with an exceptionally late onset of the disease (10 
years) and was EBV negative. EBV-positive PTLD cases tend to occur earlier (median interval 6-10 months) than EBV-negative cases (median interval 4-5 years), which are also more likely to be of monomorphic-type or frank lymphoma. PTLD are associated with EBV reactivation in a setting of decreased host T-cell immune surveillance, leading to EBV-infected B-lymphocyte proliferation with eventual malignant lymphoma.

The majority of EBV infections that occur after transplantation, especially in adults, are clinically silent reactivations. This leads to a subsequent delay in the diagnosis of PTLD. Studies have shown a positive correlation between the degree of EBV DNAemia after transplantation and the development of PTLD, which has significant implications for the importance of monitoring viral load after transplantation. In a study done by Holman et al. [6], the risk of PTLD in viremic patients significantly increased with the peak quantity of EBV DNAemia. EBV DNAemia occurred after transplantation in significantly more SOT recipients than HSCT patients. Correspondingly, more SOT patients also developed PTLD than did HSCT patients, and also significantly later than HSCT recipients. The median length of time between transplant and diagnosis of PTLD for SOT patients is 2.8 years versus 121 days for HSCT patients. Since the occurrence of PTLD is significantly related to the viral load, constant monitoring and quantification of EBV-DNA load is utilized as a prognostic marker for the development of PTLD [6-8].

Our patient presented with an extremely rare, aggressive Burkitt's lymphoma/leukemia-PTLD. Although the majority of monomorphic PTLD fall into the category of DLBCL, other types of lymphoma may also occur. These include low-grade B-cell lymphoma, plasma cell myeloma, Hodgkin's lymphoma or Hodgkin's lymphomalike PTLD as well as various T-cell lymphomas with or without EBV infection. Our case showed morphological and immunophenotypical characteristics of Burkitt's lymphoma, with $t(8 ; 14)$ involving rearrangement of MYC gene $[9,10]$.

Compared to the general population, organ transplant patients have a 30-60-fold increased risk of developing non-Hodgkin's lymphoma. Burkitt's lymphoma after organ transplantation is often found at extranodal sites; it involves the central nervous system in immunocompromised patients more frequently than it does in immunocompetent patients. In 70\% of Burkitt's lymphoma occurring after organ transplantation, genes or gene products related to EBV can be demonstrated within the tumor cells. The EBV status of the tumor is of important prognostic significance: EBVpositive Burkitt's lymphoma occurring in organ transplant patients usually responds well to reduction or cessation of immunosuppressive therapy; in some cases permanent complete remissions can be achieved even without chemotherapy. In contrast, EBV-negative Burkitt's lymphoma has a very poor prognosis and hardly responds even to aggressive chemotherapy protocols [11]. Most cases of PTLD occur within the first year after transplantation. Late-onset PTLD, as seen in our patient, is often associated with more monoclonal lesions and consequently, carries a worse prognosis. The term PTLD represents a spectrum of B-cell hyperproliferative states that can be classified as early lesions and polymorphic and monomorphic PTLD, all of which are associated with EBV [1]. This final category of monomorphic PTLD is further divided into Burkitt's lymphoma/Burkitt's-like lymphoma, DLBCL and classic Hodgkin's-like lymphoma. Our case contrasts the rare occurrence of Burkitt's lymphoma as PTLD with more common DLBCL as PTLD. The two can be differentiated by histopathology and immunohistochemistry techniques. The typical immunophenotype of Burkitt's lymphoma is IgM+/CD10+/bcl-2-/bcl-6+ with a Ki-67 
proliferation index $>95 \%[12,13]$. All cases of Burkitt's lymphoma, in both immunocompetent and immunosuppressed hosts, have a myc gene translocation [9].

Treatment for PTLD includes pharmacologic, biologic, and newly emerging cellbased therapies. Antiviral therapies such as acyclovir and ganciclovir have commonly been used to treat PTLD. While they effectively decrease viral shedding in early-onset EBV infections, there is insufficient evidence to show that they are effective against the latent phase of EBV, namely the development of PTLD [14]. Additionally, intravenous immunoglobulins have also been used, but their efficacy against PTLD remains unknown. Immunosuppression reduction (IR) therapy remains the current first-line treatment for aggressive PTLD, such as the monoclonal lymphomas seen in our patient. The degree of IR is dependent upon the probable severity of acute graft rejection. Earlyonset PTLD patients tend to have a better outcome than those who present with lateonset PTLD. In aggressive PTLD refractory to treatment, immunosuppression is discontinued altogether [14]. For localized PTLD, surgical resection and/or radiation therapy are viable options. However, in view of diffuse proliferation of infected B cells, chemotherapy for advanced PTLD is similar to treatment of lymphoma with CHOP-like chemotherapy.

Immunotherapy with anti-B-cell antibodies is now surfacing as a first-choice therapy after the failure of IR therapy alone to achieve complete remission. In a case reported by Miyagi et al. [15], complete remission of an intra-graft PTLD was achieved through the use of rituximab along with IR therapy and chemotherapy without graft loss. It was suggested that the immunosuppressive effects of rituximab may have partially compensated for the detrimental impact of IR therapy on graft function. This complementary role of rituximab is what allowed for the IR therapy during chemotherapy. IR, antiviral medications or CMV-immunoglobulin for EBV prevention, immunosuppression withdrawal, anthracycline-based chemotherapy for control of localized or systemic clonal disease or resection of primary PTLD are various strategies for the treatment of PTLD [16]. Overall, chemotherapy has been shown to have a response rate of $70 \%$ in the treatment of malignant PTLD [17]. The current recommendation for treating PTLD, whether it is the rare Burkitt's lymphoma variation or common DLBCL, EBV-positive or EBV-negative, is a sequential combination of rituximab and chemotherapy. In conclusion, further investigations are needed to better understand the role of EBV infection in the pathogenesis of the different forms of PTLD in immunosuppressed patients.

\section{Disclosure}

The authors of this paper have no conflicts of interest to disclose. 


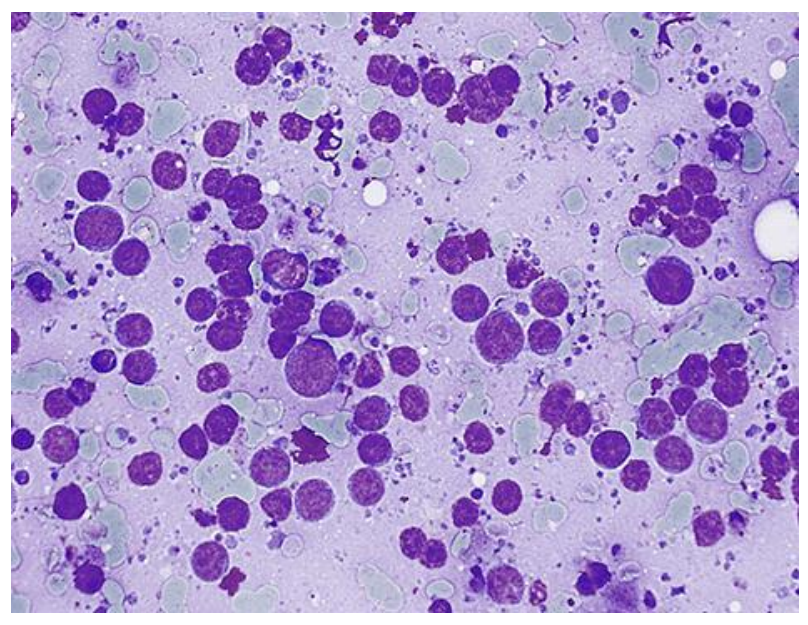

Fig. 1. Medium-sized cells with speckled chromatin and multiple small conspicuous nucleoli.

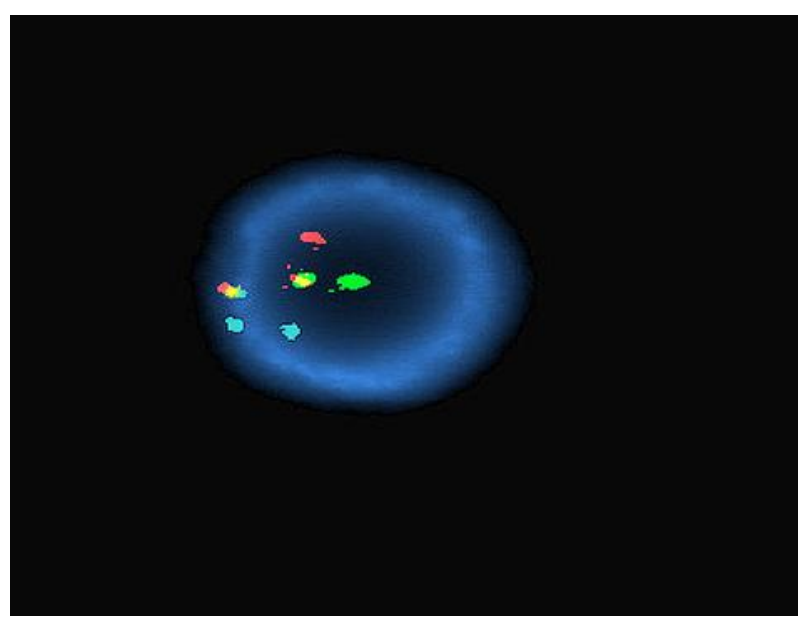

Fig. 2. Molecular cytogenetic analysis revealed a significant number of MYC/IgH fusion events. 


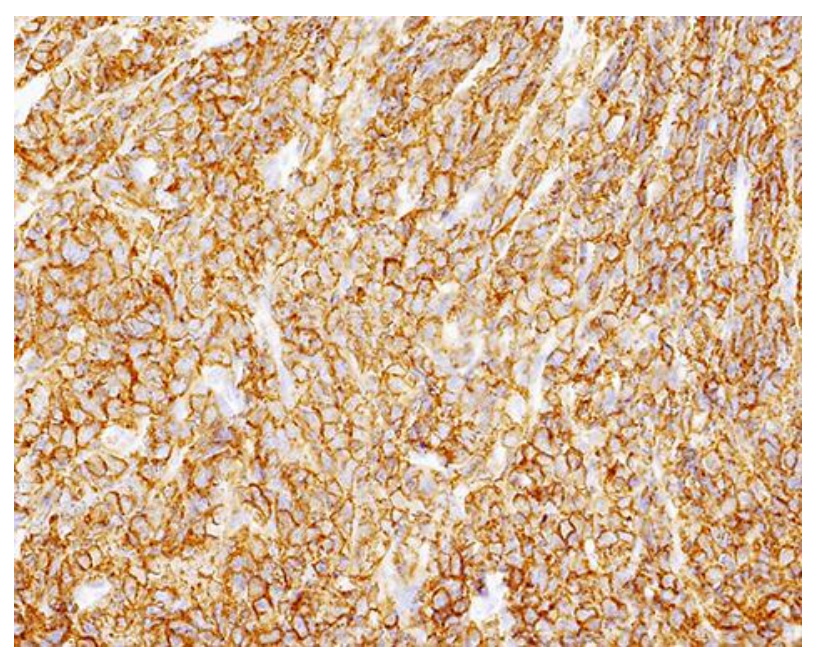

Fig. 3. Tumor cells are strongly positive for CD20, consistent with mature B-cell lineage.

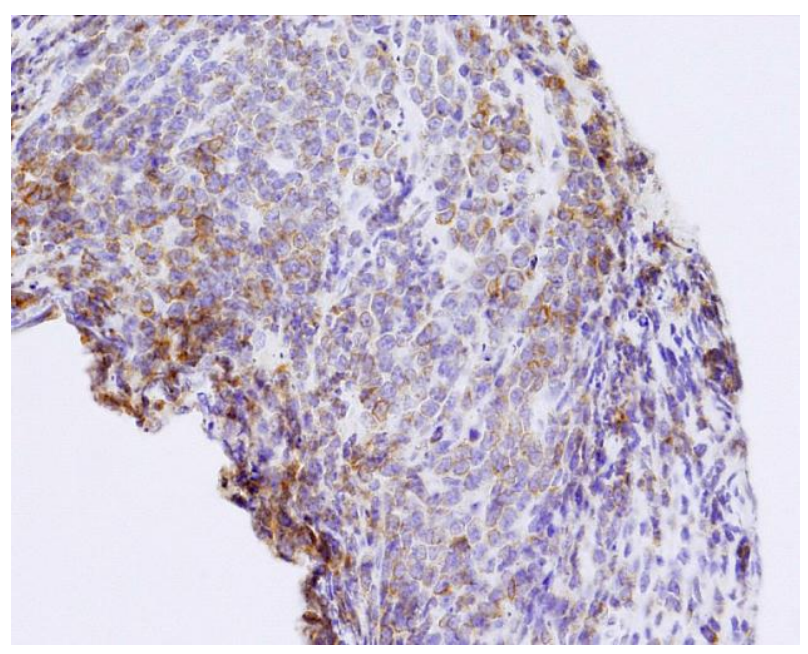

Fig. 4. Tumor cells show weak expression of BCL-2. 


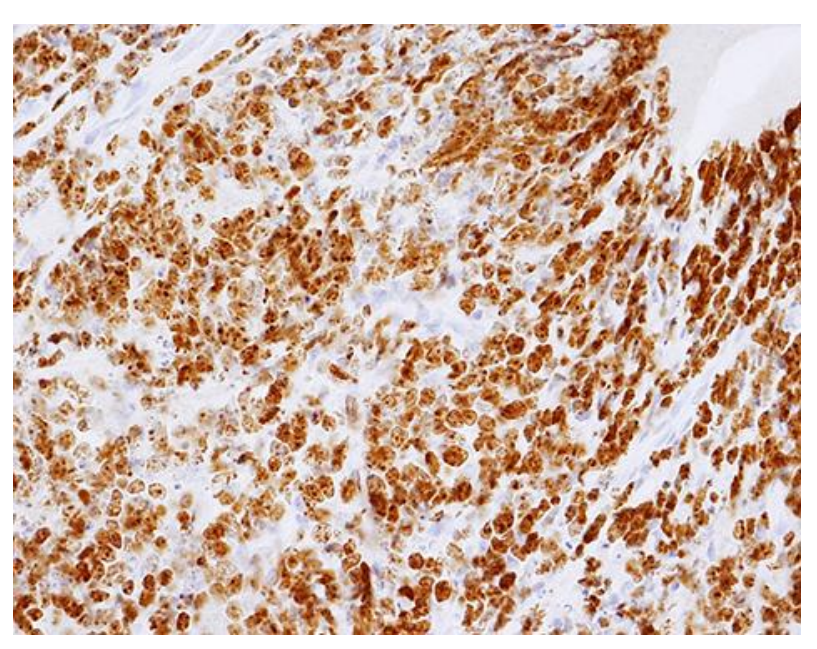

Fig. 5. Proliferation index is $100 \%$ by Mib-1 immunohistochemistry.

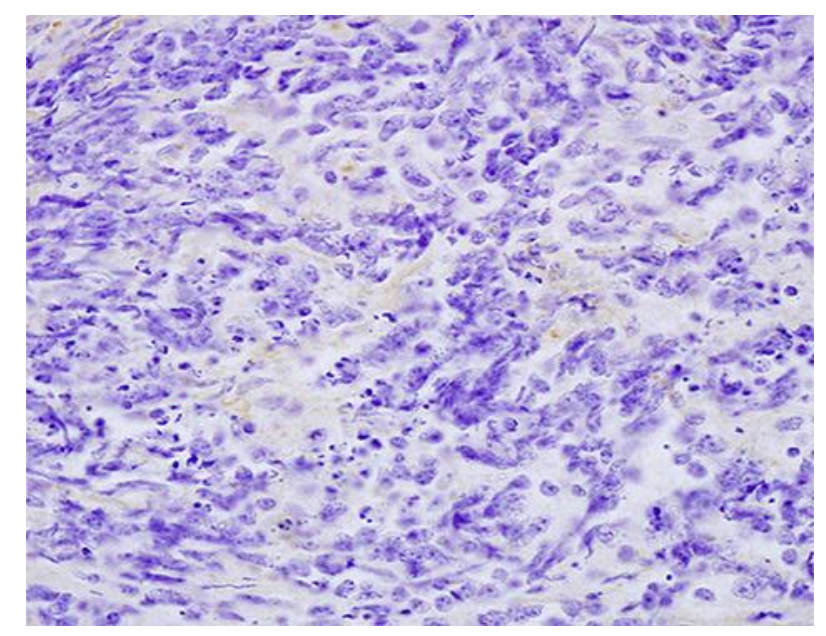

Fig. 6. EBV by in situ hybridization is negative in this tumor.

\section{References}

1 Carbone A, Gloghini A, Dotti G: EBV-associated lymphoproliferative disorders: classification and treatment. Oncologist 2008;13:577-585.

2 Pasquale MA, Weppler D, Smith J, Icardi M, Amador A, Gonzalez M, et al: Burkitt's lymphoma variant of post-transplant lymphoproliferative disease (PTLD). Pathol Oncol Res 2002;8:105-108.

3 Durand-Panteix S, Farhat M, Youlyouz-Marfak I, Rouaud P, Ouk-Martin C, David A, et al: B7-H1, which represses EBV-immortalized B cell killing by autologous T and NK cells, is oppositely regulated by c-Myc and EBV latency III program at both mRNA and secretory lysosome levels. J Immunol 2012;189:181190.

4 Pervez S, Raza MQ, Mirza A, Pal A: Strong BCL2 expression in Burkitt lymphoma is not uncommon in adults. Indian J Pathol Microbiol 2011;54:290-293.

5 Ghigna MR, Reineke T, Rince P, Schuffler P, El McHichi B, Fabre M, et al: Epstein-Barr virus infection and altered control of apoptotic pathways in posttransplant lymphoproliferative disorders. Pathobiology 2013;80:53-59. 
-6 Holman CJ, Karger AB, Mullan BD, Brundage RC, Balfour HH Jr: Quantitative Epstein-Barr virus shedding and its correlation with the risk of post-transplant lymphoproliferative disorder. Clin Transplant 2012;26:741-747.

-7 Merlino C, Cavallo R, Bergallo M, Giacchino F, Bollero C, Negro Ponzi A, et al: Epstein-Barr viral load monitoring by quantitative PCR in renal transplant patients. New Microbiol 2003;26:141-149.

-8 Orii T, Ohkohchi N, Kikuchi H, Koyamada N, Chubachi S, Satomi S, et al: Usefulness of quantitative realtime polymerase chain reaction in following up patients with Epstein-Barr virus infection after liver transplantation. Clin Transplant 2000;14(4 Pt 1):308-317.

-9 Nakamura N, Nakamine H, Tamaru J, Nakamura S, Yoshino T, Ohshima K, et al: The distinction between Burkitt lymphoma and diffuse large B-Cell lymphoma with c-myc rearrangement. Mod Pathol 2002;15:771-776.

10 Knowles DM: The molecular genetics of post-transplantation lymphoproliferative disorders. Springer Semin Immunopathol 1998;20:357-373.

11 Leblond V, Davi F, Charlotte F, Dorent R, Bitker MO, Sutton L, et al: Posttransplant lymphoproliferative disorders not associated with Epstein-Barr virus: a distinct entity? J Clin Oncol 1998;16:2052-2059.

12 Chuang SS, Ye H, Du MQ, Lu CL, Dogan A, Hsieh PP, et al: Histopathology and immunohistochemistry in distinguishing Burkitt lymphoma from diffuse large B-cell lymphoma with very high proliferation index and with or without a starry-sky pattern: a comparative study with EBER and FISH. Am J Clin Pathol 2007;128:558-564.

13 Picarsic J, Jaffe R, Mazariegos G, Webber SA, Ellis D, Green MD, et al: Post-transplant Burkitt lymphoma is a more aggressive and distinct form of post-transplant lymphoproliferative disorder. Cancer 2011;117:4540-4550.

14 Hatton 0, Martinez OM, Esquivel CO: Emerging therapeutic strategies for Epstein-Barr virus+ posttransplant lymphoproliferative disorder. Pediatr Transplant 2012;16:220-229.

15 Miyagi S, Sekiguchi S, Kawagishi N, Akamatsu Y, Satoh K, Takeda I, et al: Rituximab therapy and reduction of immunosuppression to rescue graft function after renal posttransplantation lymphoproliferative disorder found by macrohematuria in a pancreas and kidney transplant recipient: a case report. Transplant Proc 2011;43:3299-3301.

16 Zimmermann H, Reinke P, Neuhaus R, Lehmkuhl H, Oertel S, Atta J, et al: Burkitt post-transplantation lymphoma in adult solid organ transplant recipients: sequential immunochemotherapy with rituximab (R) followed by cyclophosphamide, doxorubicin, vincristine, and prednisone (CHOP) or R-CHOP is safe and effective in an analysis of 8 patients. Cancer 2012;118:4715-4724.

-17 Trappe R, Riess H, Babel N, Hummel M, Lehmkuhl H, Jonas S, et al: Salvage chemotherapy for refractory and relapsed posttransplant lymphoproliferative disorders (PTLD) after treatment with single-agent rituximab. Transplantation 2007;83:912-918. 